\title{
The Role of Imaging in Hypertensive Heart Disease
}

\author{
Edward T. D. Hoey, FRCR ${ }^{1}$ Vijayabhaskar Pakala, FRCR ${ }^{1}$ Jun K. Teoh, MRCP ${ }^{2}$ Helen Simpson, MRCP ${ }^{2}$ \\ ${ }^{1}$ Department of Radiology, Heart of England NHS Foundation Trust, \\ Birmingham, United Kingdom \\ 2 Department of Cardiology, Heart of England NHS Foundation Trust, \\ Birmingham, United Kingdom

\begin{abstract}
Address for correspondence Edward T. D. Hoey, FRCR, Department of Radiology, Heart of England NHS Foundation Trust, Heartlands Hospital, Bordesley Green East, Birmingham, West Midlands, United
\end{abstract} \\ Kingdom B9 5SS (e-mail: edwardhoey1@gmail.com).
}

Int ] Angiol 2014;23:85-92.

\begin{abstract}
Keywords

- echocardiography

- magnetic resonance imaging

- hypertension

Hypertensive heart disease (HHD) describes a spectrum of target organ response that includes left ventricular hypertrophy, systolic, and diastolic dysfunction. A variety of imaging techniques can be used to assess the various aspects of HHD. Echocardiography has for many years been the main imaging technique in the evaluation of HHD, but there is an increasing role for cardiovascular magnetic resonance (CMR) imaging due to its ability to provide an unrestricted field of view and noninvasive tissue characterization. This article reviews the current role of imaging for HHD with particular focus on echocardiography and CMR applications.
\end{abstract}

Hypertensive heart disease (HHD) describes a spectrum of target organ response that includes left ventricular hypertrophy (LVH), systolic, and diastolic dysfunction and their clinical manifestations such as heart failure and arrhythmias. ${ }^{1}$ The presence of hypertension confers a twofold increased risk of heart failure in men and threefold increased risk in women as well as more than doubling the risk of acute myocardial infarction and stroke. ${ }^{2}$ As well as the heart, longstanding hypertension affects other target organs including the kidneys, eyes, and peripheral arteries.

The classical paradigm of HHD is LVH as a compensatory response to minimize wall stress followed by left ventricular dilation and a gradual decline in ejection fraction leading to dilated heart failure. ${ }^{1}$ There is however considerable interindividual variability in the adaptive response including the pattern of LVH which is thought to be due to many interrelated factors including differences in pressure loading, concomitant medical conditions (such as coronary artery disease), genetic influences, and the neurohormonal response. ${ }^{1}$ It is also well recognized that patients with HHD can develop diastolic dysfunction and/or the clinical syndrome of heart failure with normal ejection fraction (HFNEF), which is associated with remodeling of the extracellular matrix and an increase in left ventricular filling pressures. $^{3}$
A variety of imaging techniques can be used to assess the various aspects of HHD including HFNEF. This article reviews the current role of imaging in this setting with particular focus on echocardiography and cardiovascular magnetic resonance (CMR) imaging.

\section{Left Ventricular Hypertrophy}

LVH is defined by an increase in LV mass qualified by measurements of postmortem weight, by electrocardiographic (ECG) criteria, and by echocardiography or CMR. Early echocardiographic studies by Devereux and Reichek defined LVH as an absolute LV mass exceeding 250 g. 4 Recently, gender-specific values indexed for age and body surface area have been published using CMR as the gold standard technique. ${ }^{5}$ There is a direct linear relationship between LV mass and cardiovascular morbidity and mortality. ${ }^{2}$ There is also evidence that regression of LV mass with antihypertensive treatment confers a lower risk of stroke, myocardial infarction, and all-cause mortality. ${ }^{6}$ The development of LVH is therefore an important step in the progression of HHD on the pathway to heart failure and has been the subject of intense study in recent years. Many pathological changes are present in patients with hypertensive LVH including enlargement of cardiac myocytes and increased 
amounts of interstitial and perivascular fibrosis. ${ }^{3}$ There are three main patterns of LVH first described by Ganau et al: concentric hypertrophy, eccentric hypertrophy, and concentric remodeling. ${ }^{7}$ Concentric LVH is when LV mass predominantly increases by wall thickening in response to pressure overload. It is more common in middle aged and elderly patients and associated with lower cardiac output. Eccentric hypertrophy is when LV mass predominantly increases by chamber dilatation. It is more common in young patients and associated with higher cardiac output. ${ }^{8}$ Concentric remodeling is when LV mass is within the normal range but there is demonstrable LVH. It is still not entirely clear why patients develop a specific LV geometric response to hypertension with factors such as pressure overload, volume overload, ethnicity, gender, obesity, and plasma renin levels all thought to have influence. ${ }^{1}$ In addition, it is unclear if the LVH pattern adds any relevant prognostic information. Although ECG measures of LVH were associated with cardiovascular disease risk in the Framingham study, ${ }^{9}$ it is generally agreed that ECG assessment of LVH lacks sensitivity and specificity, particularly in young male patients. ${ }^{10}$

\section{Echocardiography}

The reported prevalence of echocardiographic LVH in patients with hypertension is approximately $40 \%{ }^{2} \mathrm{M}$-mode echocardiography was the first method to be validated for LV mass assessment. It provides excellent endocardial definition and is usually quick and simple to perform. However, it only measures the LV in one dimension and assumes an ellipsoid shape with uniform wall thickness throughout the LV. As such Mmode derived measures have been shown to have low accuracy and have been progressively supplanted by measurements made using two-dimensional (2D) techniques. ${ }^{11}$ 2D echocardiography allows more accurate and reproducible measurements of LV volumes and mass and is mostly commonly calculated using the Devereux equation. ${ }^{12}$ It should be noted that this formula is applicable only to patients without major distortion of their left ventricular geometry (e.g., left ventricular aneurysm):

LV $\quad$ mass $=0.8 \times\left(1.04 \quad\left[(\text { LVIDd }+ \text { PWTd }+ \text { SWTd })^{3}-\right.\right.$ $\left.\left.(\text { LVIDd) })^{3}\right]\right)+0.6 \mathrm{~g}$

where LVIDd is the left ventricular internal diameter in diastole, PWTd is the posterior wall thickness in diastole, and SWTd is the septal wall thickness in diastole.

For patients with an increased LV mass, calculation of relative wall thickness (RWT) using the equation $(2 \times$ PWTd/LVIDd) will aid in classification of concentric or eccentric LVH. Patients with an increased RWT (RWT $\geq 0.42$ ) have concentric hypertrophy, whereas patients with a normal value (RWT $\leq 0.42$ ) have eccentric hypertrophy. Patients with concentric remodeling are those with normal LV mass but increased RWT. Echocardiography derived LV mass and RWT measurements have been shown to carry prognostic significance in hypertensive patients, even those without LVH. $^{13}$ Three-dimensional (3D) echocardiography is a relatively new technique which has several advantages over Mmode and 2D methods as it does not rely on geometric assumptions. ${ }^{14}$ It is highly reproducible for assessment of
LV mass and volumes but as with any echocardiography technique relies on adequate acoustic windows and an experienced practitioner.

\section{Cardiovascular Magnetic Resonance}

CMR is considered the reference technique for quantifying LV mass, wall thickness, and dimensions with the highest accuracy and reproducibility of all noninvasive techniques. ${ }^{5}$ Multiple short axis cine "slices" using a bright blood prepared steady state-free-precession (SSFP) sequence are acquired from the mitral valve annulus to the apex. From this dataset, $\mathrm{LV}$ volumes and mass are calculated by defining and summing the endocardial and epicardial contours in end systole and end diastole. As such, no geometric assumptions are needed and body habitus is not a limitation which makes this technique accurate even in those with distorted LV geometry. ${ }^{5}$ Because of excellent contrast resolution between the blood pool and myocardium provided with the SSFP sequence, the endocardial and epicardial contours are easy to define and ventricular volumes and mass can be assessed with a variance in the order of $5 \%$ which far surpasses that of echocardiography. ${ }^{15}$ For this reason, studies using CMR measures of LV mass and volumes usually require much smaller cohorts of patients due to more accurate detection of small degrees of change ( - Fig. $\mathbf{1}$ ).

\section{Differentiation of Other Causes of Left Ventricular Hypertrophy}

HHD is just one of many causes of LVH with others including hypertrophic cardiomyopathy (HCM), Anderson-Fabry disease, sarcoidosis, amyloidosis, aortic stenosis, and athlete's heart. In many cases, the distinction from hypertensionrelated $\mathrm{LVH}$ will be straightforward based on a combination of clinical features and key imaging determinates, namely, LVH distribution, $\mathrm{LV}$ function, and the presence and pattern of CMR detected myocardial fibrosis (-Fig. 2). Myocardial fibrosis is a common end point of many diseases including HHD and can be imaged noninvasively using a gadolinium enhanced inversion recovery CMR technique. Rudolph et al recently showed that around $50 \%$ of patients with hypertension and LVH had patchy mid-wall late gadolinium enhancement which is thought to reflect fibrosis-related expansion of the extracellular space. ${ }^{16}$

HHD and HCM might occasionally be difficult to differentiate, but in general, LV wall thickness of HHD is less pronounced (15-16 mm) and usually uniform in distribution. Although HCM can give symmetric LV thickening, more often it produces an asymmetric pattern and is often associated with cavity obliteration and/or signs of outflow tract compromise such as systolic anterior mitral valve leaflet motion. A patchy late gadolinium enhancement pattern can be seen with both HCM and HHD although is usually more pronounced in the former. ${ }^{17}$

Andersson-Fabry disease (an X-linked recessive lysosomal storage disorder) causes progressive myocardial accumulation of globotriaosylceramide leading to LVH and congestive cardiac failure. CMR studies have shown that gadolinium enhancement within the basal inferolateral wall is specific 


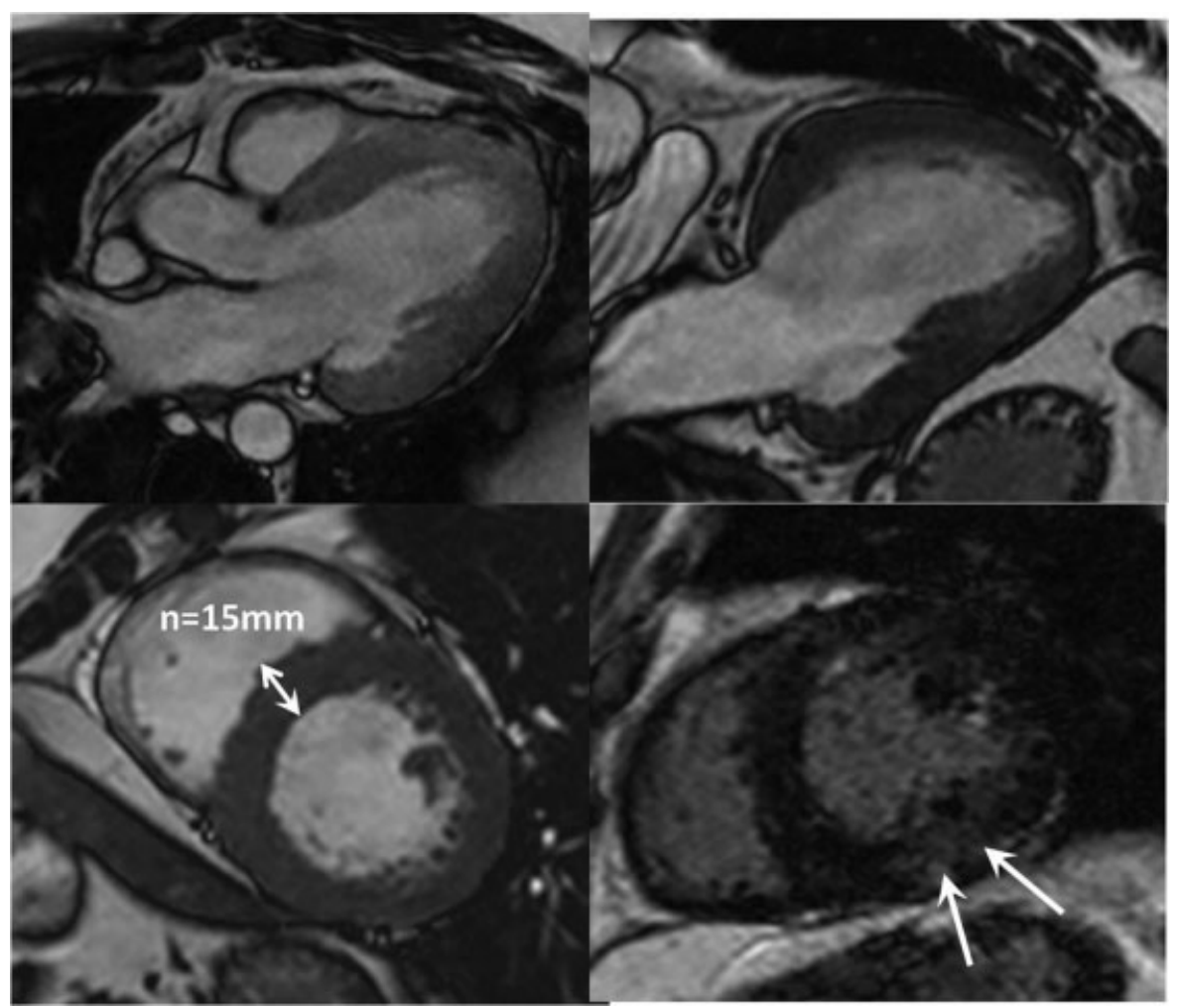

Fig. 1 Hypertensive heart disease assessed with CMR. There is global myocardial left ventricular hypertrophy demonstrated on three-chamber, two-chamber, and mid-left ventricle short axis SSFP images. Bottom right late gadolinium-enhanced image shows subtle patchy mid-wall fibrosis (arrows). CMR, cardiovascular magnetic resonance; SSFP, steady state-free-precession.

\section{Hypertrophic Cardiomyopathy}

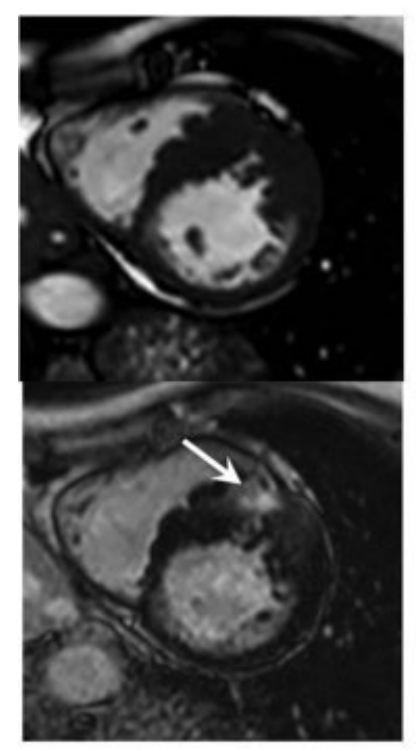

Fabry's Disease

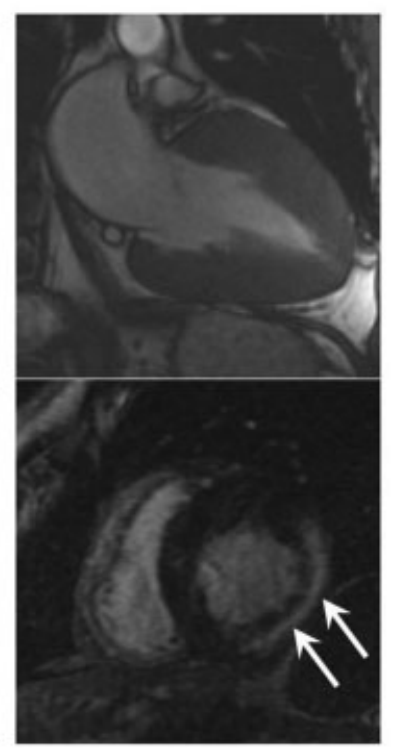

Cardiac Amyloidosis

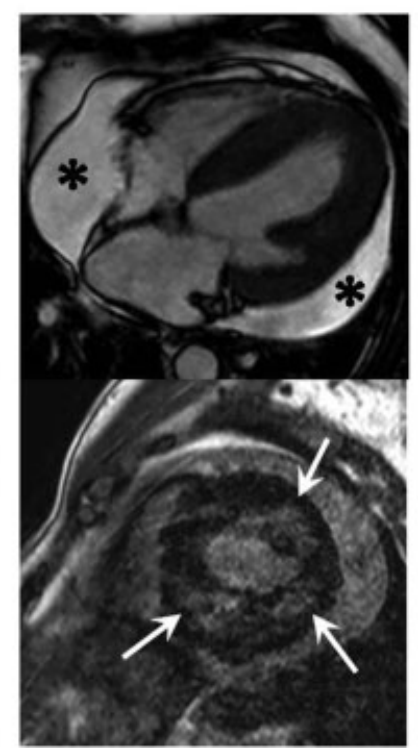

\section{Cardiac} Sarcoidosis

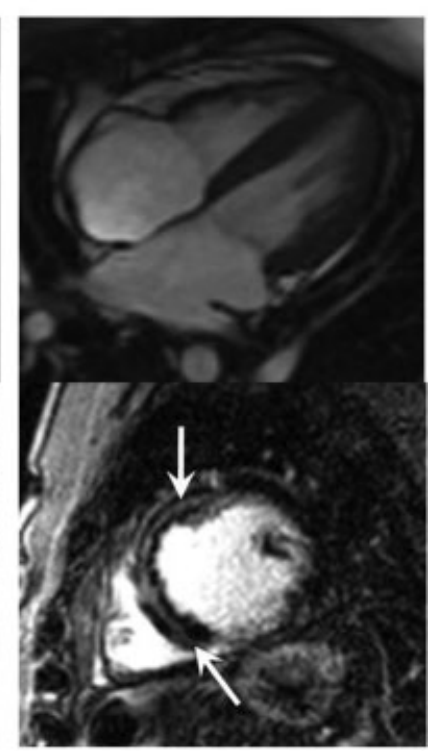

Fig. 2 Differential patterns of LVH assessed with CMR. Diastolic SSFP cine images (top row) with corresponding late gadolinium enhancement images (bottom row) illustrating a variety of causes of left ventricular hypertrophy and some specific patterns of enhancement which aid with diagnosis. Hypertrophic cardiomyopathy images showing asymmetric hypertrophy of the mid anteroseptum with corresponding patchy intramyocardial fibrosis (arrow). Fabry disease images showing pronounced circumferential hypertrophy with a prominent focus of mid-wall fibrosis within the basal inferolateral wall (arrows). Amyloidosis images showing circumferential hypertrophy with a moderate-sized pericardial effusion (asterisks) and patchy subendocardial circumferential fibrosis (arrows). Sarcoidosis images showing mild hypertrophy and prominent focus of mid-wall fibrosis along the interventricular septum (arrows). CMR, cardiovascular magnetic resonance; LVH, left ventricular hypertrophy; SSFP, steady state-free-precession. 
to Fabry disease as patients with LVH of other causes have variable locations and distributions of gadolinium enhancement. $^{18}$

Cardiac sarcoidosis classically shows LVH with gadolinium enhancement localized in the basal and subepicardial myocardium although other patterns have been described. ${ }^{19}$ Often, there will be other supportive imaging signs to aid with differentiation such as mediastinal lymphadenopathy and lung parenchymal distortion.

Amyloid heart disease usually displays typical features of a restrictive cardiomyopathy with $\mathrm{LVH}$, diastolic dysfunction, and disproportionate atrial enlargement. Other common features include thickening of the interatrial septum and presence of a small volume pericardial effusion. ${ }^{20}$ In cardiac amyloidosis, gadolinium chelates distribute in the interstitial space that is expanded by amyloid infiltration. This leads to a distinctive pattern of global subendocardial gadolinium enhancement. The blood pool signal is unusually low in patients with amyloid heart disease likely reflecting high tissue uptake and rapid washout from blood pool. The diffuse nature of enhancement in this disease is usually a reliable discriminator from sarcoidosis, HCM, and HHD. ${ }^{21}$ Recently, CMR has been shown to be useful in assessing prognosis through detailed analysis of myocardial gadolinium kinetics although this technique is still limited to highly specialized CMR units. ${ }^{22}$

Morphologic adaptations of an athlete's heart may mimic HHD, HCM, and dilated cardiomyopathy. Typically, there is mild LVH (usually $<16 \mathrm{~mm}$ ) and increased left ventricular volumes but no evidence of diastolic dysfunction. A diastolic wall thickness divided by the left ventricular end-diastolic volume ratio of less than $0.15 \mathrm{~mm} / \mathrm{m}^{2} / \mathrm{mL}$ as defined by Petersen et al is regarded as the best parameter to differentiate an athlete's heart from all other pathologic causes of $\mathrm{LVH}^{23}$ Another important feature of athletic remodeling is a lack of late gadolinium enhancement.

\section{Assessment of Global and Regional Left Ventricular Systolic Function}

Hypertension is known to alter myocardial mechanics through a complex chain of adaptive responses. The net result is reduced myocardial deformation which may initially be regional but in the longer term can affect global LV function and prognosis. Transthoracic echocardiography can assess LV mid-wall shortening (MWS) as an indirect measurement of myocardial function and has been shown to be depressed in patients with HHD. ${ }^{24}$ Echocardiographic assessment of MWS cannot at present distinguish regional variations in function as it is a geometry-based index derived from both posterior and septal wall measurements. CMR can also be used to measure MWS via a tissue tagging technique which monitors displacement of defined myocardial tissue points through the cardiac cycle (-Fig. $\mathbf{3}$ ). CMR has the advantage of providing both regional and global strain information. Rosen et al have recently shown a strong correlation between high diastolic blood pressure and regional LV dysfunction. ${ }^{25}$ The main current drawback of tissue tagged CMR is the complex and often time-consuming postprocessing techniques required.

\section{Assessment of Left ventricular Diastolic Function}

Patients with HHD are at increased risk of developing diastolic dysfunction (which describes abnormal mechanical properties) and/or HFNEF (which describes the clinical syndrome) otherwise known as HFNEF. At echocardiography, HFNEF patients have a normal LV ejection fraction but clinically they have signs and symptoms of heart failure. Up to $50 \%$ of hypertensive patients have been shown to have some imaging features of diastolic dysfunction. ${ }^{26}$ In patients on treatment for hypertension, diastolic function has been shown to improve as LVH regresses. $^{27}$

\section{Echocardiography}

A variety of echocardiography methods can be used to characterize diastolic function. Measuring left atrial (LA) volume is a fundamental step as in the absence of mitral valve disease; LA dilatation is reliable marker of diastolic dysfunction, providing a direct reflection of elevated left ventricular filling pressure. Effective medical treatment of hypertension has been shown to reduce LA volume in parallel with regression of $\mathrm{LVH}$ and reduction in left ventricular end-

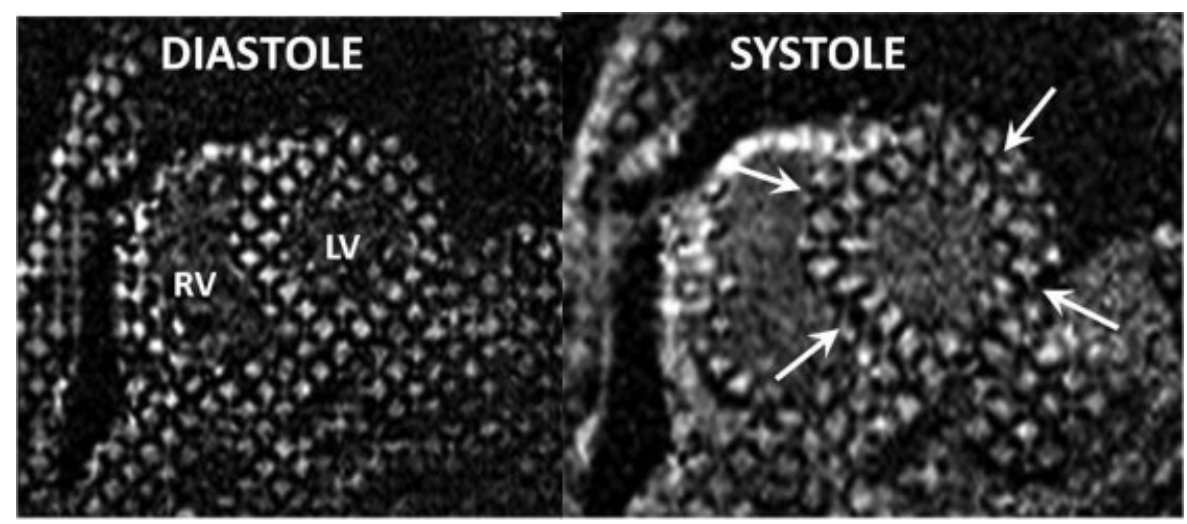

Fig. 3 Myocardial tagging. Left ventricular short axis cine MRI images in diastole and systole acquired using the myocardial tagging technique. Grid lines across the left ventricular myocardium can be tracked through the cardiac cycle and their degree of deformation (arrows) used to calculate regional and global shortening. CMR, cardiovascular magnetic resonance; LV, left ventricle; MRI, magnetic resonance imaging; RV, right ventricle. 
diastolic pressure. ${ }^{28}$ LA volumes can be measured by 2D methods using the biplane area-length method from apical four- and two-chamber views, or by 3D echocardiography. Indexing to body size is derived by dividing LA volume by body surface area $\left(\mathrm{m}^{2}\right)$. An LA volume of $\geq 34 \mathrm{mLm}^{2}$ has been shown to be an independent predictor of cardiovascular complications. ${ }^{29}$ Isovolumetric ventricular relaxation time (IVRT) is measured as the period between aortic valve closure and mitral valve opening. IVRT is a good screening tool for approximating the LA pressure with a shortened IVRT $(<60$ ms) considered significant.

In early hypertension there is mild "stiffening" and delayed relaxation of the myocardium which affects peak early filling (E-wave) and late diastolic filling (A-wave). This manifests as a reduced E/A ratio of transmitral inflow velocities. Pulsed wave Doppler is used to record mitral inflow velocity at the tips of the mitral leaflets. Mild diastolic dysfunction manifests as a reduced $\mathrm{E} / \mathrm{A}$ ratio and with progressive diastolic dysfunction there is pseudonormalization of the $\mathrm{E} / \mathrm{A}$ ratio and subsequently a high E/A ratio representing restrictive physiology. ${ }^{30}$

Tissue Doppler imaging (TDI) is a relatively newer technique which is of value in assessment of diastolic dysfunction. TDI enables direct quantification of mitral annular flow velocities with a reduced diastolic mitral annular velocity suggesting diastolic dysfunction. Unlike conventional Doppler assessment, TDI values can be corrected for changes in preload or heart rate. $^{31}$

Another emerging echocardiography technique is 2Dspeckle imaging which has the potential to detect very early alterations in myocardial velocity and regional function. Speckle imaging gives assessment of circumferential, longitudinal, and radial LV strain patterns and has been proposed as a promising technique for detection of subclinical dysfunction in hypertensive patients who might benefit from early prevention. For example, speckle tracking-derived longitudinal strains are reduced in hypertension with preservation of circumferential and radial strains which may be an early marker of altered LV mechanics. ${ }^{32}$

\section{Cardiovascular Magnetic Resonance}

Diastolic function can also be measured with CMR similar to the methods used in Doppler echocardiography. CMR phase contrast sequences (which induce phase shifts in moving protons that are directly proportional to their velocity) can be acquired across the mitral valve to yield information on early and late left ventricular filling patterns. The addition of pulmonary vein systolic, early diastolic, and atrial systolic peak flow velocities as well as longitudinal myocardial early and late diastolic flow velocities obtained at the base of the heart can improve evaluation of diastolic function. ${ }^{33}$ Studies have shown good agreement between CMR and echocardiography derived diastolic inflow velocity measurements.

Myocardial fibrosis is associated with increased ventricular stiffness, impairment of LV systolic function, and development of diastolic dysfunction. ${ }^{3}$ The extent of myocardial fibrosis as quantified by gadolinium-enhanced CMR has also been shown to directly correlate with the severity of diastolic dysfunction in patients with hypertension. ${ }^{34}$ Recently, there has been much interest in the use of novel CMR sequences such as T1-mapping to more sensitively detect and characterize the extent and distribution of myocardial fibrosis. T1-mapping amplifies regional variations in gadolinium distribution making it easier to detect diffuse myocardial fibrosis compared with standard inversion recovery CMR pulse sequences.

\section{Assessment for Secondary Causes of Systemic Hypertension}

Secondary causes of hypertension include renal arterial disease, aortic coarctation, and pheochromocytoma. The prevalence of these conditions varies widely and the requirement for imaging is guided by clinical suspicion and failure to achieve a target blood pressure response with optimal antihypertensive drug treatment. In many cases, CMR will be the imaging technique of choice owing to its unrestricted field of view, lack of ionizing radiation, and excellent soft tissue resolution.

Renal artery stenosis (RAS) is estimated to account for up to $35 \%$ of patients with resistant hypertension. The commonest form is atherosclerotic which typically involves the proximal portion of the renal artery and may be associated with renal atrophy. Fibromuscular dysplasia is much less common, mainly affects the mid and distal vessel and predominantly affects younger female patients. Gadolinium-enhanced CMR angiography has a sensitivity and specificity of greater than $90 \%$ for detection of RAS. ${ }^{35}$

Aortic coarctation is a narrowing of the aortic lumen which can occur in two distinct types. Adult coarctation is characterized by an abrupt stenosis of a short segment of the postductal thoracic aorta, usually occurring just distal to the left subclavian artery origin. In contrast, the infantile form is characterized by diffuse hypoplasia of the arch. In around $20 \%$ of those affected, the disorder is diagnosed in adulthood with recognized complications including systemic hypertension, intracranial aneurysm rupture, and bicuspid aortic valve degeneration. The exact mechanism whereby hypertension occurs is unclear with theories including activation of the renin-angiotensin system and neurohormonal changes. Transthoracic echocardiography with good acoustic windows may be able to establish the diagnosis and quantify any pressure gradient, but CMR is usually indicated for a complete overview of anatomy including the presence of collateral supply and functional assessment of hemodynamic compromise (-Fig. 4). ${ }^{36}$

Pheochromocytoma is a catecholamine-secreting tumor which may precipitate life-threatening hypertension or cardiac arrhythmias. They are rare, occurring in 0.1 to $0.9 \%$ of hypertensive individuals. More than $90 \%$ are located within the adrenal glands, and $98 \%$ occur within the abdomen. Extraadrenal pheochromocytomas develop in paraganglionic tissue of the sympathetic nervous system anywhere from the base of the brain to the urinary bladder. They are characteristically solid, hypervascular masses but a wide spectrum of appearances have been described owing to variable 


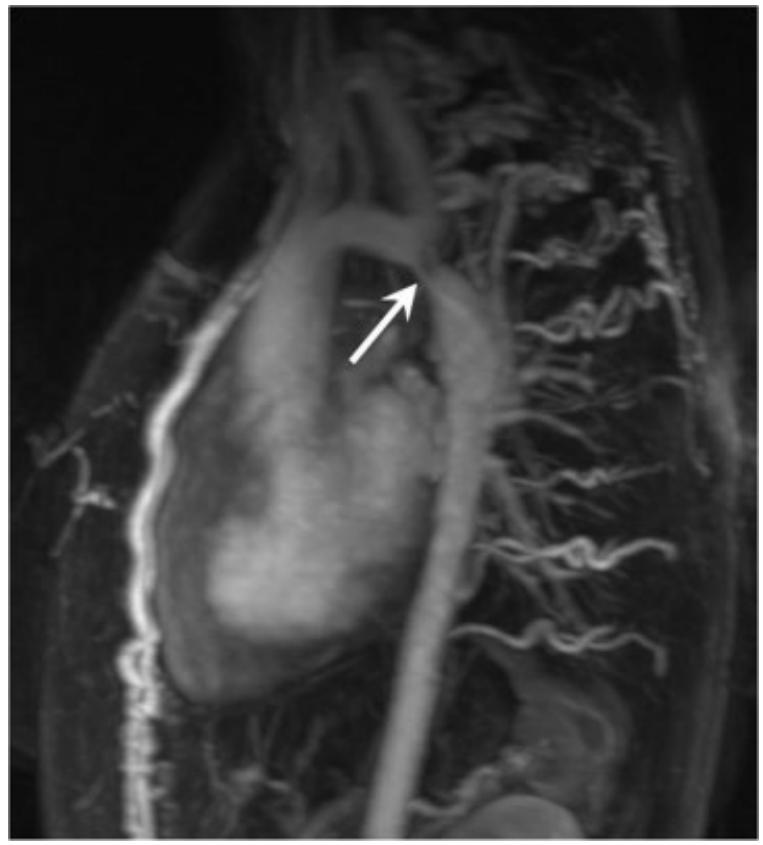

Fig. 4 CMR of aortic coarctation. Sagittal thick section reconstruction from a gadolinium-enhanced thoracic aorta angiogram showing a severe focal narrowing of the proximal descending thoracic aorta in keeping with a tight aortic coarctation. Note the multiple enlarged intercostal and internal mammary vessels that help bypass the obstruction. CMR, cardiovascular magnetic resonance.

compositions of tumor tissue, calcifications, and blood products. The most common magnetic resonance imaging appearance of pheochromocytoma is an adrenal mass with low signal intensity on T1-weighted imaging and high signal intensity on T2-weighted imaging although this is not a universal finding as up to a third of cases may have low signal intensity on T2-weighted imaging. ${ }^{37}$

\section{Conclusion}

Advances in echocardiography and CMR techniques over the last decade have provided new insights into the morphological and pathophysiological changes associated with HHD. Comprehensive assessment of systolic and diastolic function provides prognostically relevant data. CMR is a particularly attractive imaging technique as it is not restrained by acoustic windows, provides the most accurate and reproducible measures of LV function and mass, and enables myocardial fibrosis assessment. In addition, CMR is the most reliable means of distinguishing hypertension-related LVH from other causes and can also be used to screen for secondary causes of systemic hypertension.

\section{References}

1 Drazner $\mathrm{MH}$. The progression of hypertensive heart disease. Circulation 2011;123(3):327-334

2 Levy D, Garrison RJ, Savage DD, Kannel WB, Castelli WP. Prognostic implications of echocardiographically determined left ventricular mass in the Framingham Heart Study. N Engl J Med 1990;322(22): 1561-1566
3 Berk BC, Fujiwara K, Lehoux S. ECM remodeling in hypertensive heart disease. J Clin Invest 2007;117(3):568-575

4 Devereux RB, Reichek N. Echocardiographic determination of left ventricular mass in man. Anatomic validation of the method. Circulation 1977;55(4):613-618

5 Alfakih K, Walters K, Jones T, Ridgway J, Hall AS, Sivananthan M. New gender-specific partition values for ECG criteria of left ventricular hypertrophy: recalibration against cardiac MRI. Hypertension 2004;44(2):175-179

6 Devereux RB, Wachtell K, Gerdts E, et al. Prognostic significance of left ventricular mass change during treatment of hypertension. JAMA 2004;292(19):2350-2356

7 Ganau A, Devereux RB, Roman MJ, et al. Patterns of left ventricular hypertrophy and geometric remodeling in essential hypertension. J Am Coll Cardiol 1992;19(7):1550-1558

8 Ross J Jr. On variations in the cardiac hypertrophic response to pressure overload. Circulation 1997;95(6):1349-1351

9 Kannel WB, Gordon T, Offutt D. Left ventricular hypertrophy by electrocardiogram. Prevalence, incidence, and mortality in the Framingham study. Ann Intern Med 1969;71(1):89-105

10 Pewsner D, Jüni P, Egger M, Battaglia M, Sundström J, Bachmann LM. Accuracy of electrocardiography in diagnosis of left ventricular hypertrophy in arterial hypertension: systematic review. BMJ 2007;335(7622):711

11 Salcedo EE, Gockowski K, Tarazi RC. Left ventricular mass and wall thickness in hypertension. Comparison of $\mathrm{M}$ mode and two dimensional echocardiography in two experimental models. Am J Cardiol 1979;44(5):936-940

12 Devereux RB, Alonso DR, Lutas EM, et al. Echocardiographic assessment of left ventricular hypertrophy: comparison to necropsy findings. Am J Cardiol 1986;57(6):450-458

13 Li L, Shigematsu Y, Hamada M, Hiwada K. Relative wall thickness is an independent predictor of left ventricular systolic and diastolic dysfunctions in essential hypertension. Hypertens Res 2001; 24(5):493-499

14 Kühl HP, Schreckenberg M, Rulands D, et al. High-resolution transthoracic real-time three-dimensional echocardiography: quantitation of cardiac volumes and function using semi-automatic border detection and comparison with cardiac magnetic resonance imaging. J Am Coll Cardiol 2004;43(11):2083-2090

15 Grothues F, Smith GC, Moon JC, et al. Comparison of interstudy reproducibility of cardiovascular magnetic resonance with twodimensional echocardiography in normal subjects and in patients with heart failure or left ventricular hypertrophy. Am J Cardiol 2002;90(1):29-34

16 Rudolph A, Abdel-Aty H, Bohl S, et al. Noninvasive detection of fibrosis applying contrast-enhanced cardiac magnetic resonance in different forms of left ventricular hypertrophy relation to remodeling. J Am Coll Cardiol 2009;53(3):284-291

17 Ho CY, López B, Coelho-Filho OR, et al. Myocardial fibrosis as an early manifestation of hypertrophic cardiomyopathy. $\mathrm{N}$ Engl J Med 2010;363(6):552-563

18 Moon JC, Sachdev B, Elkington AG, et al. Gadolinium enhanced cardiovascular magnetic resonance in Anderson-Fabry disease. Evidence for a disease specific abnormality of the myocardial interstitium. Eur Heart J 2003;24(23):2151-2155

19 Smedema JP, Snoep G, van Kroonenburgh MP, et al. Evaluation of the accuracy of gadolinium-enhanced cardiovascular magnetic resonance in the diagnosis of cardiac sarcoidosis. J Am Coll Cardiol 2005;45(10):1683-1690

20 Falk RH, Dubrey SW. Amyloid heart disease. Prog Cardiovasc Dis 2010;52(4):347-361

21 Maceira AM, Joshi J, Prasad SK, et al. Cardiovascular magnetic resonance in cardiac amyloidosis. Circulation 2005;111(2): 186-193

22 Maceira AM, Prasad SK, Hawkins PN, Roughton M, Pennell DJ. Cardiovascular magnetic resonance and prognosis in cardiac amyloidosis. J Cardiovasc Magn Reson 2008;10:54-65 
23 Petersen SE, Selvanayagam JB, Francis JM, et al. Differentiation of athlete's heart from pathological forms of cardiac hypertrophy by means of geometric indices derived from cardiovascular magnetic resonance. J Cardiovasc Magn Reson 2005;7(3):551-558

24 Shimizu G, Hirota Y, Kita Y, Kawamura K, Saito T, Gaasch WH. Left ventricular midwall mechanics in systemic arterial hypertension. Myocardial function is depressed in pressure-overload hypertrophy. Circulation 1991;83(5):1676-1684

25 Rosen BD, Saad MF, Shea S, et al. Hypertension and smoking are associated with reduced regional left ventricular function in asymptomatic: individuals the Multi-Ethnic Study of Atherosclerosis. J Am Coll Cardiol 2006;47(6):1150-1158

26 Redfield MM, Jacobsen SJ, Burnett JC Jr, Mahoney DW, Bailey KR, Rodeheffer RJ. Burden of systolic and diastolic ventricular dysfunction in the community: appreciating the scope of the heart failure epidemic. JAMA 2003;289(2):194-202

27 Smith VE, White WB, Meeran MK, Karimeddini MK. Improved left ventricular filling accompanies reduced left ventricular mass during therapy of essential hypertension. J Am Coll Cardiol 1986;8(6):1449-1454

28 Mattioli AV, Bonatti S, Monopoli D, Zennaro M, Mattioli G. Influence of regression of left ventricular hypertrophy on left atrial size and function in patients with moderate hypertension. Blood Press 2005;14(5):273-278

29 Tsang TS, Abhayaratna WP, Barnes ME, et al. Prediction of cardiovascular outcomes with left atrial size: is volume superior to area or diameter? J Am Coll Cardiol 2006;47(5):1018-1023
30 Ishida Y, Meisner JS, Tsujioka K, et al. Left ventricular filling dynamics: influence of left ventricular relaxation and left atrial pressure. Circulation 1986;74(1):187-196

31 Nagueh SF, Middleton KJ, Kopelen HA, Zoghbi WA, Quiñones MA. Doppler tissue imaging: a noninvasive technique for evaluation of left ventricular relaxation and estimation of filling pressures. J Am Coll Cardiol 1997;30(6):1527-1533

32 Narayanan A, Aurigemma GP, Chinali M, Hill JC, Meyer TE, Tighe DA. Cardiac mechanics in mild hypertensive heart disease: a speckle-strain imaging study. Circ Cardiovasc Imaging 2009; 2(5):382-390

33 Chai P, Mohiaddin R. How we perform cardiovascular magnetic resonance flow assessment using phase-contrast velocity mapping. J Cardiovasc Magn Reson 2005;7(4):705-716

34 Moreo A, Ambrosio G, De Chiara B, et al. Influence of myocardial fibrosis on left ventricular diastolic function: non-invasive assessment by CMR and echo. Circ Cardiovasc Imaging 2009;2(6): 437-443

35 Vasbinder GB, Nelemans PJ, Kessels AG, Kroon AA, de Leeuw PW, van Engelshoven JM. Diagnostic tests for renal artery stenosis in patients suspected of having renovascular hypertension: a metaanalysis. Ann Intern Med 2001;135(6):401-411

36 Hom JJ, Ordovas K, Reddy GP. Velocity-encoded cine MR imaging in aortic coarctation: functional assessment of hemodynamic events. Radiographics 2008;28(2):407-416

37 Blake MA, Kalra MK, Maher MM, et al. Pheochromocytoma: an imaging chameleon. Radiographics 2004;24(Suppl 1):S87-S99 